\title{
Research on the Design Theory of Swirl Generator Attached to a New Bidirectional Flowmeter
}

\author{
Li Huazhi, Liang Qianchao \\ College of Architecture and power \\ Naval University of Engineering \\ Wuhan, China
}

\author{
Cui Hanguo, Zhang Shun \\ College of Architecture and power \\ Naval University of Engineering \\ Wuhan, China
}

\begin{abstract}
Based on the application of hydrodynamics, a new method of designing the swirl generator attached to a new bidirectional flowmeter is put forward. The 3-dimensions simulation in CFD is carried out to study the fluid motion for various amount of vanes attached to the swirl generator and various intake velocities. The results of simulation testify that the tangential velocity is linear proportional to radius according to the new design method for the swirl generator which can be used in a new bidirectional flowmeter. The simulation results also show the reverse flow exist $s$ in lower intake velocity whereas increasing the amount of vanes attaching to the swirl generator will lessen the reverse flow. The method of simulation in 3-Dimensions model in this paper will be an example in fluid analysis. The 3-D fluid simulation for annular cylinder will do good to study the similar field. The results will be applied in designing the swirl generator and measuring the cubage flux by the new flowmeter.
\end{abstract}

Keywords- Swirl generator, Flowmeter, Simulation analysis, 3-Dimensions fluid

\section{INTRODUCTION}

A new method of designing the swirl generator attached to a new swirl flowmeter is put forward. The swirl generator attaching to the flowmeter is the most important unit which influent the metering error. Many people have studied the swirl flows basing on the combustors or jets[1-7].

Batterson[1] reviewed some of the theoretical solutilons used to describe swirl dominated flows in both unidirectional and bidirectional flow orientations, and then he illuminated all swirl dominated flows comprise a forced vortex core centered around their axis of rotation. The core is due to viscous forces and increases in size with successive increases in viscosity. It is delineated by the point where the swirl velocity reaches its maximum. Within the forced vortex core, the tangential velocity is linearly proportional to the radius, a characteristic of rigid body rotation. Outside the forced vortex core, the tangential velocity gradually becomes inversely proportional to the radius, thus exhibiting a free vortex tail. Sheen[2] developed an experimental investigation to derive a new correlation for the swirl number of a radial-type swirl generator under various Reynolds numbers and various vane angle conditions. Three-component velocity profiles of axial, radial, and azimuthal components at the swirling jet exit were measured for various flow conditions. Based on the experimental results, a modified swirl number $\mathrm{S}$ is derived to characterize the swirling flow. Alekseenko[3] studied the effects of external periodical axisymmetric forcing on the jet flow structure and considered the greatest effect of the forcing on the swirling jet structure was observed in the case of the high swirl rate $(\mathrm{S}=1.0)$. Basing on impinging jet Alekseenko[4] also developed an experimental investigation using advanced pre- and post-processing algorithms to study the influence of swirl rate on the flow structure. It was found that the magnitude of pressure diffusion decreased with the growth of the swirl rate. Xia [5] developed a numerical and experimental study of strongly swirling flow in a water model combustion chamber equipped with a swirler of special design. He considered the differential Reynolds stress model (DRSM) captures all the major features of the swirling flow whereas the RNG k-e model or the standard k-e model take for the swirling flow downstream as a solid-body-rotation-type flow. Basing on impinging annular jet Thomas[6] has studied the performance of heat transfer with Large Eddy Simulation (LES) for three different swirl numbers which corresponding range from non-swirling to strongly swirling flow. Buckley [7] provided a design method for Integral Rocket and Ramjets conformed to Carter's rule states. All above scholars have obtained beneficial conclusions for the design of swirl generator except for simulation in 3-Dimensions for annular passage. Basing on the design the fluid in hollow cylindric measure pipe is analyzed and a new design method of the swirl generator is deduced. Simulation in CFD will be carried out to study the fluid motion in annular pipe.

\section{CONFIGURATION AND MEASURE PRINCIPLE}

The configuration of the new swirl flowmeter is designed as Figure.1.

The system consists of columniform pipe, middle shaft, vane of steady flow and the swirl generator which all are fixed on middle shaft, signal transformer and annular path, ball with high magnetoconductivity. The flow section between two concentric circles is perpendicular to the flow axis which is also the axis of pipe. Two vanes and two swirl generators are fixed symmetrically about the symmetrical axis of configuration in order to realize the function of bidirectional measurement. The signal transformer consists of a sensor with an electromagnetic winding and a detector of frequency. The fluid can level off after passing the vane of steady flow and produce a swirl after passing the swirl 
generator. Driving by the swirl flow, the ball designed to have the approximate density with the measured liquid can circumvolve following the annular path. The sensor can produce a series of pulse signal because of magnetic induction and the detector will take count of the running frequency of ball. Similarly to the turbine flowmeter, the flowrate can be measured after transforming the signal of frequency in processor.

\section{FLUID IN MEASURE PIPE ANALYSIS}

The fluid in pipe moves as follow hypotheses:

(a) The fluid comprising the swirl after the swirl generator moves symmetrically about the flow axis. The fluid moves in steady state which doesn't change along with time. Analyzing the fluid in cylindric pipe in cylindrical coordinates, the following equations can be found:

$$
\begin{aligned}
& \frac{\partial}{\partial t}=0 \\
& \frac{\partial V_{\theta}}{\partial \theta}=\frac{\partial V_{r}}{\partial \theta}=\frac{\partial V_{z}}{\partial \theta}=\frac{\partial p}{\partial \theta}=0
\end{aligned}
$$

In this paper, $V_{\theta}, V_{r}$ and $V_{z}$ are respectively the velocity in the tangential direction, the radial direction and the axial direction.

(b) The fluid is incompressible and the influence of gravity is ignored.

(c)The density of the ball with high magnetoconductivity approximate to that of measured fluid.

From the configuration of the flowmeter, the above hypotheses are appropriate.

Basing on the above hypotheses, the continuity equation and the Navier-Strokes equations in cylindrical coordinates are given as follows:

$$
\frac{\partial V_{r}}{\partial r}+\frac{\partial V_{z}}{\partial z}+\frac{V_{r}}{r}=0
$$

$$
\left.\begin{array}{l}
V_{r} \frac{\partial V_{\theta}}{\partial r}+V_{z} \frac{\partial V_{\theta}}{\partial z}+\frac{V_{r} V_{\theta}}{r}=v\left(\frac{1}{r} \frac{\partial V_{\theta}}{\partial r}+\frac{\partial^{2} V_{\theta}}{\partial r^{2}}+\frac{\partial^{2} V_{\theta}}{\partial z^{2}} \frac{V_{\theta}}{r^{2}}\right) \\
V_{r} \frac{\partial V_{r}}{\partial r}+V_{z} \frac{\partial V_{r}}{\partial z} \frac{V_{\theta}^{2}}{r}=-\frac{1}{\rho} \frac{\partial p}{\partial r}+v\left(\frac{1}{r} \frac{\partial V_{r}}{\partial r}+\frac{\partial^{2} V_{r}}{\partial r^{2}}+\frac{\partial^{2} V_{r}}{\partial z^{2}} \frac{V_{r}}{r^{2}}\right) \\
V_{r} \frac{\partial V_{z}}{\partial r}+V_{z} \frac{\partial V_{z}}{\partial z}=-\frac{1}{\rho} \frac{\partial p}{\partial z}+v\left(\frac{1}{r} \frac{\partial V_{z}}{\partial r}+\frac{\partial^{2} V_{z}}{\partial r^{2}}+\frac{\partial^{2} V_{z}}{\partial z^{2}}\right)
\end{array}\right\}
$$

Here, $v$ and $\rho$ are respectively the viscosity and density of measured liquid, $p$ is the fluid pressure of one point in pipe. Basing on the conservation of mass principle and the density is constant; the following equation can be deduced:

$$
\rho \cdot \bar{V}_{1} \cdot t \cdot s_{1}=\rho \cdot \bar{V}_{2} \cdot t \cdot s_{2}
$$

Here, $\bar{V}_{1}$ and $S_{1}$ are respectively the average velocity and the area of intake section, $V_{2}$ and $S_{2}$ are respectively the average velocity and the area of outlet section.

The swirl generator is unwrapped into straight cascade at $r_{a v}$ which is the average of the outer radius of the swirl generator and the wheel boss as Figure. 2.

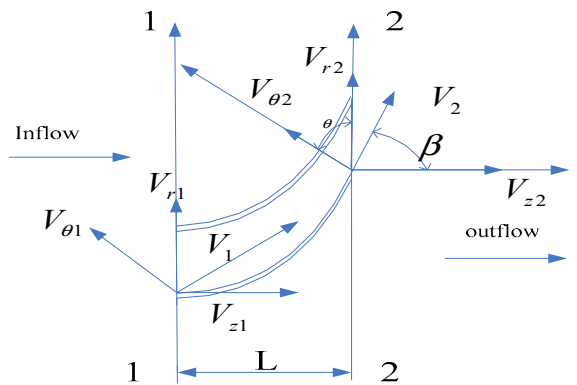

Figure 2. The velocity triangle in straight cascade

The initial velocity at inlet section is axial along with the flow axis which $V_{r}$ and $V_{\theta}$ are zero. Here the value of inlet is labeled subscript " 1 " and the value of outlet is labeled subscript " 2 ". $\beta$ is the fixed angle between the cascade of the swirl generator at $r_{a v}$ and the flow axis.

The area of intake section of measure pipe is equal to that of outlet section. $\bar{V}$ is the average velocity of fluid in pipe.Therefore, $S_{1}=S_{2}$. The equation (5) can be written as:

$$
\bar{V}_{1=} \bar{V}_{2}
$$
follow:

From equation (6), the equation (7) can be supposed as

$$
\frac{\partial V_{z}}{\partial z}=0
$$

Simultaneous equations (3) and (7), the following equation can be obtained:

$$
\frac{\partial V_{r}}{\partial r}+\frac{V_{r}}{r}=0
$$

From equation (8), $V_{r}$ can be obtained by equation (9):

$V_{r}=\frac{C_{1}}{r} \quad\left(C_{1}\right.$ is a constant $)$

Supposing the vanes of the swirl generator are very thin 
cascades and designed to keep the equal fixed angle of $\beta$ in any position, the fluid movement is close to the axisymmetric flow which keep fixed outlet angle of $\beta$ after the swirl generator[8] as Figure.2. After the section of inflow, the following equation (10) can be obtained:

$$
\left.\begin{array}{l}
\sqrt{V_{\theta}^{2}+V_{r}^{2}}=V_{z} \tan \beta \\
\tan \beta=\frac{r_{a v}}{L}\left(\frac{2 \pi}{N}+\frac{\pi \alpha}{180}\right) \\
r_{a v}=\frac{r_{H}+r_{B}}{2}
\end{array}\right\}
$$

Where, $r_{H}$ and $r_{B}$ is respectively the outer radius and the wheel boss of the swirl generator. $L$ is the axial length of the swirl generator. $N$ is the amount of vane on the swirl generator. $\alpha$ is the overlap angle among the adjacent vane. $r_{a v}$ which is designed to be the average of $r_{H}$ and $r_{B}$ is to keep the vane of the swirl generator symmetrical about the axial section at $r_{a v}$, which the outlet and the inlet of the swirl generator can be interconverted in bidirectional measure.

The intensity of swirl flow is usually characterized by the swirl number. The definition of the swirl number is based on the geometry of swirl generator [9] :

$$
S=\frac{2}{3}\left(\frac{1-\left(r_{B} / r_{H}\right)^{3}}{1-\left(r_{B} / r_{H}\right)^{2}}\right) \tan \beta
$$

Here, $\beta$ is not chosen as the airflow turning angle [7] which is designed to be compatible with typical ramjet configurations.

Simultaneous equations (7), (9) and (10), equation (4) can be transformed into:

$$
\left.\begin{array}{l}
\frac{\partial^{2} V_{\theta}}{\partial r^{2}}+\frac{v-C_{1}}{r v} \frac{\partial V_{\theta}}{\partial r}-\frac{v+C_{1}}{r^{2} v} V_{\theta}=0 \\
\frac{1}{\rho} \frac{\partial p}{\partial r}=\frac{C_{1}^{2}}{r^{3}}+\frac{\mathrm{V}_{\theta}^{2}}{r} \\
\frac{1}{\rho} \frac{\partial p}{\partial z}=v \frac{\partial^{2} V_{z}}{\partial r^{2}}+\frac{v-C_{1}}{r} \frac{\partial V_{z}}{\partial r}
\end{array}\right\}
$$

When the vane of swirl generator is designed to be radial direction at any point, $V_{r}$ is close to be zero and then $C_{1}$ is zero too. Therefore, the following equation (13) can be obtained from the first equation of equation (11):

$$
V_{\theta}=\frac{C_{2}}{r}+\omega r
$$

Where, $C_{2}$ and $\omega$ are determined by the movement of fluid in pipe. In theory, when $r$ is zero, $V_{\theta}$ is infinity. Considering the tangential velocity $V_{\theta}$ is finite. $C_{2}$ is chosen to be zero, the flow behind the swirl generator is similar to the forced vortex. Equation (13) can be expressed as follow:

$$
V_{\theta}=\omega r=2 \pi f r
$$

Here, $\omega$ is the angle velocity of the fluid behind the swirl generator. $f$ is the rotation frequency of the fluid behind the swirl generator. $f$ can be measured as the rotation frequency of the ball in middle pipe.

Simultaneous equations (10), (12) and (14), the following equations can be obtained:

$$
\left.\begin{array}{l}
V_{z}=\frac{\omega r}{\tan \beta} \\
\frac{\partial p}{\partial r}=\rho \omega^{2} r \\
\frac{\partial p}{\partial z}=\frac{\omega \rho v}{r \tan \beta}
\end{array}\right\}
$$

\section{A Model}

\section{SIMULATION AND ANALYSIS}

The swirl generators whose vanes are almost radial direction at any point are designed as figure 3 . The parameters of swirl generator in figure 3(a) are: $N=4$, ${ }^{r} H=0.046 \mathrm{~m}, \quad r_{B}=0.014 \mathrm{~m}, \quad L=0.036 \mathrm{~m}, \quad \alpha=20^{\circ}, \mathrm{S}=1.14$. The parameters of swirl generator in figure 3(b) are: $N=5$, $r_{H}=0.046 \mathrm{~m}, \quad r_{B}=0.014 \mathrm{~m}, \quad L=0.036 \mathrm{~m}, \alpha=20^{\circ}, \mathrm{S}=0.9554$. The parameters of swirl generator in figure 3(c) are: $N=6$, ${ }^{r} H=0.046 \mathrm{~m}, \quad r_{B}=0.014 \mathrm{~m}, \quad L=0.036 \mathrm{~m}, \alpha=20^{\circ}, \mathrm{S}=0.83$. All are strongly swirling flow.

$B$ Simulation

According to above deductions, the flowrate is characterized as the motion behind the swirl generator. After the swirl generator, accompanied with the vortex the recirculation zone and associated region of high turbulence [10]] will influence the motion of the ball which can rotate around the annular path. According to equation (16), the inlet pressure will exert influence on the fluid motion in pipe. Next, following various swirl numbers $S$ and the parameters of the swirl generator, simulations in CFD will be carried out to study the fluid motion and the influence on the measure performances.

For torus flow of hollow cylinder, Reynolds numbers is 
given by

$$
R_{e}=\frac{\bar{V}\left(2 r_{B}-2 r_{H}\right)}{v}
$$

The medium measured is water in 20 degree centigrade. From equation (21), when $\left.\bar{V}_{i n}>0.1 \mathrm{~m} / \mathrm{s}, R_{e}\right\rangle 3185$. Commonly, $\bar{V}_{i n}>0.1$, the flow in pipe is always the turbulence with high

$R_{e}$. So the arithmetic of numerical simulation is chosen as Reynolds Stress equation Model (RSM).

1) The influence of inlet velocity

Here we choose the model is Figure. 3(b). Owing to the symmetrical configuration of the flowmeter, the field in the middle of two vanes of the swirl generator is simulated which is plotted as Figure. 4(a) and the whole model is plotted as Figure. 4(b). In order to study the flow motion after the swirl generator, the simulative field after the swirl generator is extended for $0.02 \mathrm{M}$ along the axis of measuring pipe. The simulated results are plotted in the faces which are aligned with the exit face at $0.036 \mathrm{~m}, 0.042 \mathrm{~m}$ in flow axis. The faces aligned with the exit face at $0.036 \mathrm{~m}, 0.042 \mathrm{~m}$ in flow axis are respectively named after face 0.036 and face 0.042 .

The intake velocity parameters $\bar{V}_{\text {in }}$ are $15 \mathrm{M} / \mathrm{s}, 10 \mathrm{M} / \mathrm{s}$, $1.84 \mathrm{M} / \mathrm{s}$ and $0.2 \mathrm{M} / \mathrm{s}$. The correlations between ${ }^{V_{\theta}}$ and $r$ are shown as Figure. 5.

The axial velocity behind the swirl generator is plotted as Figure.6 which shows the reverse flow exists at low intake velocity. The axial velocity behind the swirl generator is plotted as Figure. 7 when $\bar{V}_{i n}=0.2 \mathrm{M} / \mathrm{s}$. The contours of velocity magnitude behind the swirl generator are plotted as Figure.8.

\section{2)The influence of the number of vanes}

Here, there are two methods of changing the number of vanes. One is to alter the amount of vane which has been designed. Another is to redesign the whole swirl generator according to equation (10) again which the structural parameters of swirl generator will change. Here the latter method is studied. The correlations between $V_{\theta}$ and $r$ which the swirl generator is redesigned for various $\mathrm{N}$ are shown as Figure. 7. When $\mathrm{N}$ is 5 , its simulated figures are plotted as Figure. 5 and Figure. 6.

\section{Discussion}

From Figure.5, the steady-time is short for all intake velocity and the tangential velocity is linear proportional to radius which conforms to equation (14). It shows the method of designing swirl generator is feasible. From Figure.6, it can be found the low intake velocity is accompanied with reverse flow.
Comparing the axial velocity behind the swirl generator in Figure. 7 with that of Figure. 6, it can be found the reverse flow exists as $\mathrm{N}=4$ at face 0.036 and face 0.042 . When $\mathrm{N}=6$, the reverse flow don't exists at face 0.036 and face 0.042 .

\section{CONCLUSION}

A new method of designing swirl generator is put forward in this paper. Various intake velocities and various number of vanes attaching to various model of swirl generator are simulated in CFD. The simulation results in CFD show the tangential velocities behind the swirl generator are linear proportional to radius which can be used in a new bidirectional flowmeter. The simulation results also show the reverse flow exist $\mathrm{s}$ in lower intake velocity whereas increasing the amount of vanes attaching to the swirl generator will lessen the reverse flow. By simulations the method of measuring flowrate by measuring the rotation frequency of ball is feasible. The method of simulation in 3-Dimensions model in this paper will be an example in fluid analysis. The 3-D fluid simulation for annular cylinder will do good to study the similar field. The results will be applied in designing the swirl generator and measuring the cubage flux by the new flowmeter.

\section{REFERENCES}

[1] Batterson J W, Maicke B A, Majdalani J. Advancements in Theoretical Models of Confined Vortex Flowfields. JANNAF Propulsion Meeting [R], 14-17 May 2007, Denver, CO.

[2] Sheen H J, Chen W J, Jeng S Y, et al. Correlation of swirl number for a radial-type swirl generator. Experimental Thermal and Fluid Science [J], 1996.12, PP444-451.

[3] Alekseenko S V, Dulin V M, Kozorezov Y S, et al. Effect of axisymmetric forcing on the structure of a swirling turbulent jet. International Journal of Heat and Fluid Flow [J], 2008.29, PP1699-1715.

[4] Alekseenko S V, Bilsky A V, Dulin V M, et al. Experimental study of an impinging jet with di?erent swirl rates. International Journal of Heat and Fluid Flow [J], 2007.28, PP1340-1359.

[5] Xia J L, Yadigaroglu G, Liu Y S, et al. Numerical and experimental study of swirling flow in a model combustor. International Journal of Heat and Fluid Flow [J], 1998. 41, PP1485-1497.

[6] Thomas H, Laszlo F. Numerical study of swirling and non-swirling annular impinging jets with heat transfer. 35th AIAA Fluid Dynamics Conference and Exhibit [C], 6-9 June 2005, Toronto, Ontario Canada.

[7] Buckley P L, Craig R R, Davis D L, et al. The design and combustion performance of practical swirlers for integral Rocket/Ramjets. ASME 16th joint propulsion conference [C], Hartford, Connecticut, 1980, June 30-July 2.

[8] Vavra M. H. Aero thermodynamics and flow in turbomachines[M]. New York: Robert E.krieger Publishing Company, 1974.

[9] Gupta, A.K., Lilley, D.G, Syred, N., Swirl Flows [M]. Abacus Press, Kent England, 1984.

[10] Lilley D G. Swirl flows in combustors. 9th AIAA fluid and plasma dynamics conference [C], 14-16 July, 1976, California, USA. 


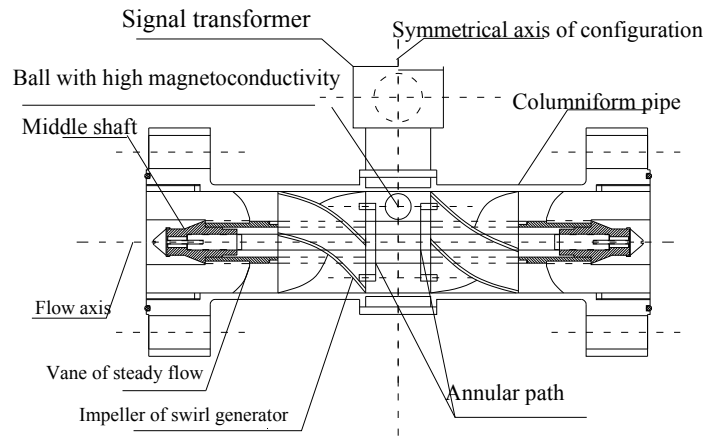

Figure 1. Configuration of the new swirl flowmeter

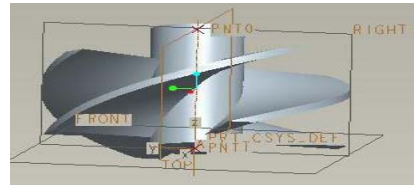

(a) $N=4$

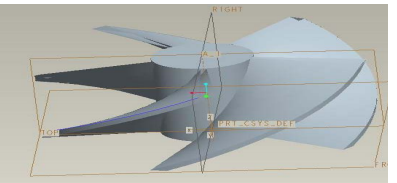

(b) $N=5$

Figure 3. Models of swirl generator

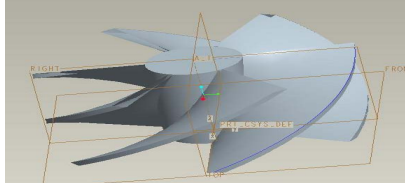

(c) $N=6$

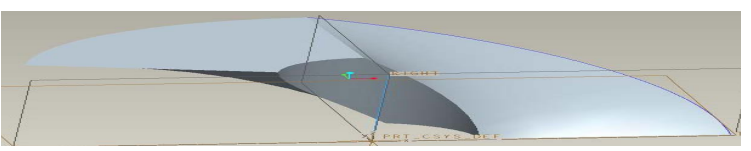

(a) One simulated field of the swirl generator

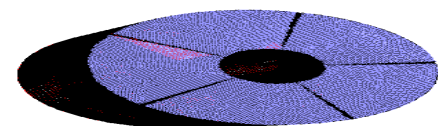

(b) The whole simulated field of the swirl generator

Figure 4. The field of simulation

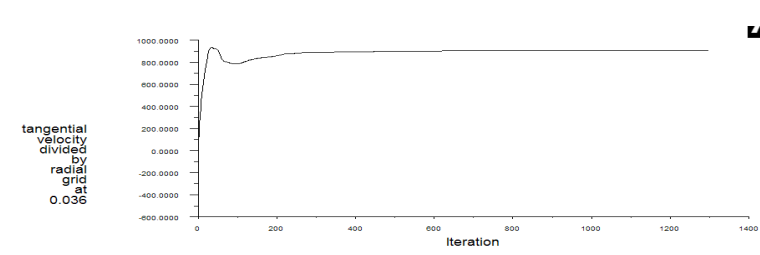

(a) $\bar{V}_{\text {in }}=15 \mathrm{M} / \mathrm{s}$

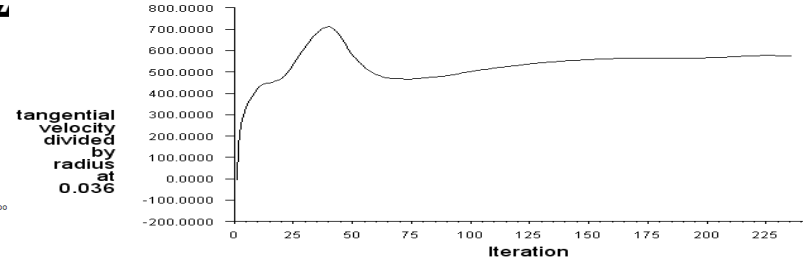

(b) $\bar{V}_{\text {in }}=10 \mathrm{M} / \mathrm{s}$
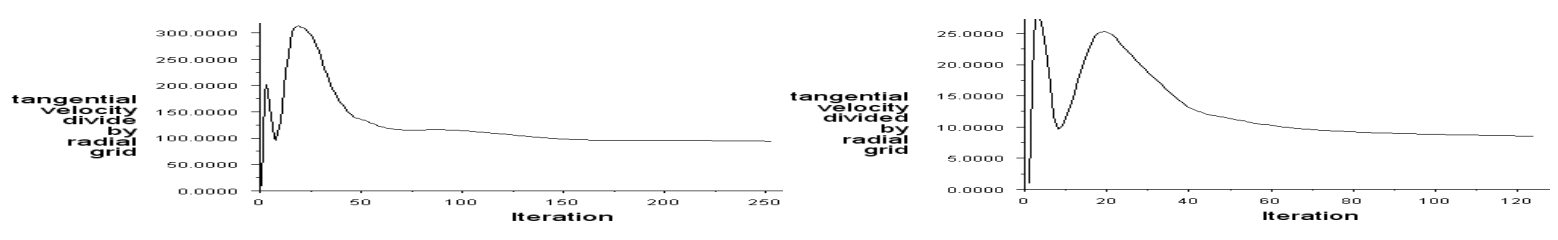

(c) $\bar{V}_{\text {in }}=1.84 \mathrm{M} / \mathrm{s}$

(d) $\bar{V}_{\text {in }}=0.2 \mathrm{M} / \mathrm{s}$

Figure 5. The correlations between $V_{\theta}$ and $r$ for various $\bar{V}_{i n}$ 

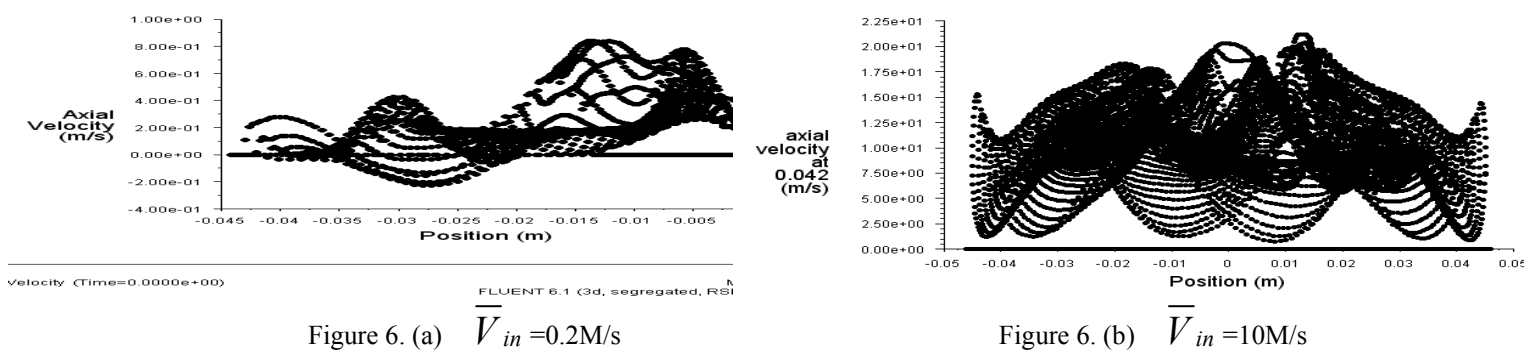

Figure 6. (b) $\bar{V}_{\text {in }}=10 \mathrm{M} / \mathrm{s}$

Figure 6. The axial velocity behind the swirl generator for various axial velocities
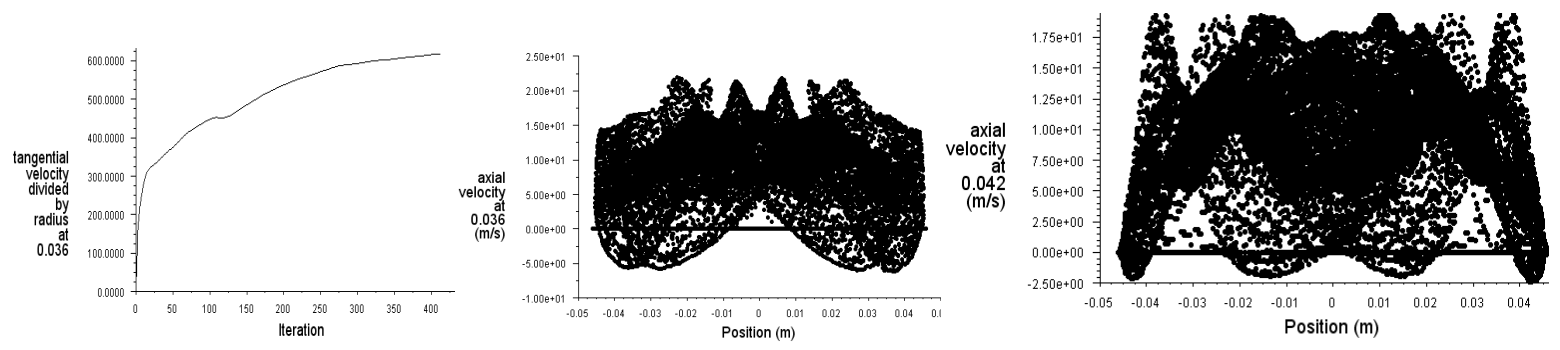

Figure 7. (a) $\mathrm{N}=4$
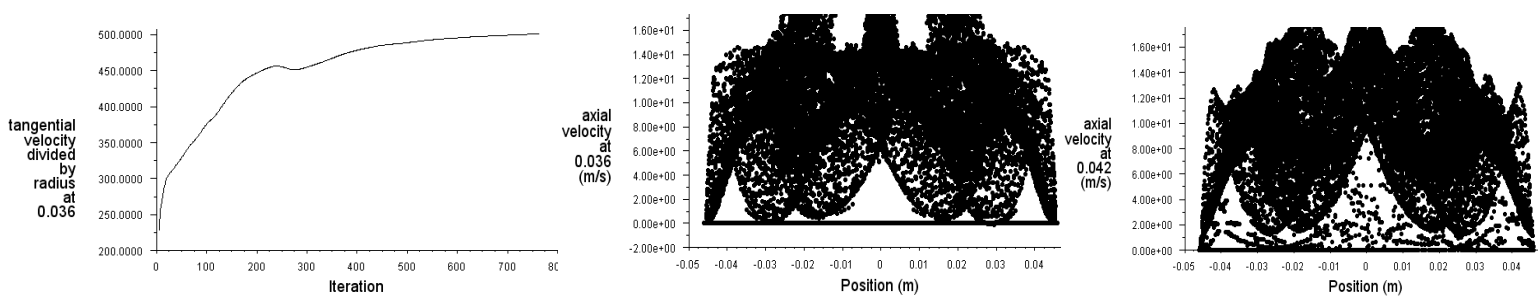

Figure 7. (b) $\mathrm{N}=6$

Figure7. The correlations between $V_{\theta}$ and $r$, the axial velocity for various $\mathrm{N}$ as $\bar{V}_{\text {in }}=10 \mathrm{M} / \mathrm{s}$ 